\title{
Perception locale de l'évolution du milieu à Oula au Nord du Burkina Faso
}

\author{
Arnaud OUEDRAOGO ${ }^{*}$, Evariste Constant Dapola DA ${ }^{2}$ et Awa Pounyala OUOBA ${ }^{2}$ \\ ${ }^{I}$ Département de Géographie, Université de Koudougou 01 BP 376 Koudougou 01/ - Burkina Faso. \\ Laboratoire Dynamiques des Espaces et Sociétés (LDES)/Université Ouaga I Pr Joseph KY-ZERBO. \\ ${ }^{2}$ Département de Géographie, Unité de Formation et de Recherche en Sciences Humaines (UFR/SH) de \\ l'Université de Ouagadougou - 03 BP 7021 Ouagadougou 03 - Burkina Faso. \\ *Auteur corresponant, E-mail : ouedrar@yahoo.fr
}

\section{RESUME}

L'évolution de l'environnement est une préoccupation majeure pour les populations locales, notamment celles des régions du Sahel. La présente étude réalisée dans la région du Nord du Burkina Faso a pour objectif l'analyse de la perception locale de l'évolution de leur environnement et des stratégies d'adaptation. La présente étude a été réalisée dans la région du Nord du Burkina Faso. La méthodologie s'est basée sur des observations de terrain, et des enquêtes socio-économiques menées auprès des populations locales sur un échantillon de 128 ménages. Les populations locales appréhendent l'évolution de leur milieu de plusieurs manières, notamment à travers la dynamique de l'occupation des terres, les changements et les variabilités climatiques ainsi que la perception des impacts sur la production agricole. Les résultats de l'étude montrent que $94 \%$ de personnes interrogées trouvent que la jachère a fortement diminué dans leur village. Aussi, $61 \%$ de répondants affirment remarquer un retard dans l'installation des pluies. Les populations locales développent des initiatives et réalisent des aménagements anti-érosifs afin de faire face à cette situation. Parmi ces réalisations anti-érosives, le zaï tient la première place (87\%), pratique la plus répandue dans l'environnement ainsi que la confection des cordons en pierres (85\%). Le compostage (92\%), les demi-lunes $(06 \%)$, etc., sont autant de techniques réalisées. Les réalisations des aménagements anti-érosifs sont d'autant plus importants au regard de la dégradation du milieu, récupérables plus facilement par les techniques antiérosives.

(C) 2016 International Formulae Group. All rights reserved.

Mots clés : Perception des populations locales, changements environnementaux, stratégie d'adaptation, Oula, Burkina Faso.

\section{Local perception of the evolution of the environment in Oula in the north of Burkina Faso}

\begin{abstract}
The development of the environment is a major concern for local populations, especially those in the Sahel regions. The purpose of this study in the Northern Region of Burkina Faso is to analyze the local perception of the evolution of their environment and adaptation strategies. The methodology was based on field observations and socio-economic surveys of local populations on a sample of 128 households. Local populations understand the evolution of their environment in a number of ways, including the dynamics of land
\end{abstract}


use, climate change and variability, and the perception of impacts on agricultural production. The results of the study show that $94 \%$ of respondents find that fallowing has declined sharply in their village. Also, $61 \%$ of respondents said they noticed a delay in the installation of the rains. Local populations are developing initiatives and implementing anti-erosion measures to deal with this situation. Among these anti-erosive achievements, the zaï holds the first place $(87 \%)$, the most widespread practice in the environment and the making of stone cords (85\%). Composting (92\%), half-moons $(06 \%)$, etc., are all techniques used. The achievements of anti-erosion installations are all the more important in view of the degradation of the environment, which can be more easily recovered by anti-erosion technics.

(C) 2016 International Formulae Group. All rights reserved.

Keywords: Perception of local populations, environmental change, adaptation strategy, Oula, Burkina Faso.

\section{INTRODUCTION}

La problématique de la variabilité du climat est devenue un enjeu majeur avec à terme, de fortes implications environnementales, sociétales et économiques (Hountondji et al., 2004; GIEC 2007; Thornton et al., 2011 ; Ouédraogo, 2015). La généralisation de la sécheresse au Sahel depuis les années 1970 représente le plus fort signal climatique en Afrique tropicale, depuis que des mesures météorologiques sont disponibles (OMM, 2001). L'assèchement climatique particulièrement prononcé qu'a connu l'Afrique de l'Ouest pendant les années 1970-1990 est allé de pair avec une transformation rapide des systèmes écologiques et sociaux (Wittig et al., 2007 ; Mahé et Paturel, 2009).

$\mathrm{Au}$ regard de cette situation peu reluisante, le GIEC (2001), déclarait que les changements climatiques observés de nos jours constituent des défis auxquels l'humanité a et aura à faire face. Les conséquences des changements climatiques sont multiples et selon l'environnement géographique où on se trouve, les phénomènes sont plus ou moins différents. Selon Barrios et al. (2008), l'impact du changement climatique touchera tous les pays du monde, avec une ampleur variable selon les régions (Tarhule, 2011). Pays sahélien de l'Afrique de l'ouest, et enclavé, du fait de sa position géographique, le Burkina Faso est particulièrement vulnérable aux changements climatiques. Dans la région soudano- sahélienne, plus particulièrement dans l'environnement local, les populations perçoivent les changements climatiques de plusieurs manières.

La commune de Oula situé au Nord du Burkina Faso, appartient au domaine soudanosahélien qui au fil du temps s'apparente plus au domaine sahélien. Le Front Inter Tropical ou FIT régit les pluies dans ce domaine climatique avec une longue saison sèche qui dure 7 à 8 mois et une courte saison de pluies dont la période varie entre 4 ou 5 mois selon les années. Les paramètres pluviométriques constituent les préoccupations majeures de cet environnement local. Ils se caractérisent par une forte variabilité des pluies dans le temps et dans l'espace. La moyenne pluviométrique est de $672,5 \mathrm{~mm} / \mathrm{an}$ pour la période de 1985 à 2014. Les températures jouent un second rôle de préoccupation pour les paysans avec une moyenne annuelle de $29,2{ }^{\circ} \mathrm{C}$ pour la période de 1984 à 2013 (Figure 5).

Le relief de la zone d'étude est plat dans l'ensemble. Le relief est légèrement perturbé par des élévations collinaires. L'altitude moyenne est comprise entre 300 et 400 mètres. La zone d'étude dispose de plusieurs types de sols dont les plus représentatifs sont les lithosols sur cuirasse, les sols ferrugineux tropicaux lessivés et les sols peu évolués d'érosion gravillonnaire (MEF, 2009).

Les formations arbustives sont caractéristiques de la région du Yatenga avec quelques reliques de savane arborée dont les 
espèces dominantes sont Parkia biglobosa, Vitellaria paradoxa, Lannea microcarpa, Guira senegalensis, Adansonia digitata, etc. Témoin d'une volonté de sauvegarde d'un environnement en déliquescence, la savane arbustive dominante composée de Balanites aegyptiaca, Detarium microcarpum, Diospiros mespiliformis, etc; avec un tapis herbacé plus ou moins continu constitué de Andropogon gayanus, Loudetia togoensis et Pennisetum pedicellatum. La végétation artificielle est composée essentiellement de Azadirachta indica, Mangifera indica et Eucalyptus camaldulensis.

A l'instar de plusieurs localités au Burkina Faso, les populations pratiquent principalement l'agriculture pluviale. Les principales cultures vivrières sont le sorgho blanc et le mil. Les activités secondaires mais lucratives sont surtout le commerce, et l'orpaillage.

L'élevage jadis de prestige est ancrée dans les mœurs. C'est une activité quasi incontournable, de par la fumure qu'il procure. Il constitue une source de revenu substantiel. Le cheptel est constitué de bovins, d'ovins, de caprins. Selon la DPRA/ Projection ENEC 2004 Yatenga, (2015) les chiffres seront en hausse jusqu'en 2025 au moins.

Partant de la position géographique dans la zone sahélienne et de ces activités économiques dépendantes de la pluviométrie pluviale, les questions suivantes peuvent être posées : a) Comment les populations locales de Oula appréhendent-elles des modifications environnementales sur leur milieu? b) et si les stratégies d'adaptation sont-elles assez efficaces?

L'objectif principal de cette étude est d'analyser la perception des populations locales de l'évolution de leur milieu à travers les changements et les variabilités climatiques.

\section{MATERIEL ET METHODES Localisation de la zone d'étude}

L'étude a été menée dans la commune de Oula située au Nord du Burkina Faso. La commune de Oula compte 62 villages administratifs et a pour chef-lieu Oula (Figure 1). Sa superficie est de $544 \mathrm{~km}^{2}$. C'est une commune qui connaît une augmentation rapide de sa population. En 1985, le recensement général de la population dénombrait 30739 habitants et 44692 habitants en 2006 (INSD, 2008). La population de Oula est composée majoritairement de Mossi et de quelques peulhs qui tentent de se sédentariser.

\section{Techniques et outils utilisés}

L'analyse des manifestations des changements climatiques à travers la perception des populations locales a nécessité diverses sources d'informations dont les observations directes sur le terrain et maintes fois opérées, des enquêtes dans trois villages. L'utilisation de données satellites a été nécessaire pour un constat scientifique cohérent. Les données climatiques de la station synoptique de Ouahigouya ont été collectées à la Direction générale de la météorologie nationale du Burkina Faso. Les paramètres pluviométriques et les autres paramètres secondaires tels que la température, dans cette étude ont concerné une période d'au moins trente ans, la période de référence selon les recommandations de l'organisation mondiale de la météorologie.

Des entretiens auprès des personnes ressources, des focus groups ainsi que des enquêtes auprès des chefs de ménages sur l'évolution de l'environnement local ont été réalisés pour vérifier notre hypothèse. $\mathrm{Au}$ regard de la spécificité des évènements relatifs au changement climatique, notamment certains phénomènes ne se produisant pas fréquemment dans le temps, les enquêtes 
auprès des chefs de ménages ont concerné essentiellement ceux ayant au moins 45 ans. A cet âge un enquêté serait à mesure de renseigner sur les évènements liés au climat trente ans auparavant au moins. Cette disposition répond au souci de recueillir des informations fiables auprès de la population cible. Au total, 128 ménages ont été enquêtés.

L'analyse de l'évolution du milieu par approche spatiale a été nécessaire pour un constat scientifique cohérent de la dynamique de l'occupation des terres. Les données d'images satellites exploitées montrent nettement les changements opérés sur l'aire d'étude. Il s'agit des images satellites Landsat de $30 \times 30$ mètres de résolution des années 1992, 2002 et 2014. Des imageries Landsat TM 195/51, 195/50, 196/51 et 196/50 qui couvrent l'environnement local ont aussi été utilisées. Les images satellites considérées ont été prises en avril, période sèche permettant d'observer nettement les surfaces cuirassées, les sols nus et les plans d'eau et d'en faire la différence nette des autres unités d'occupation des terres telles que la végétation. $\mathrm{La}$ télédétection joue un rôle important dans la gestion de la surface terrestre en fournissant des informations spatio-temporelles sur l'utilisation des terres, la couverture Zoungrana et al. (2015). Les mois de septembre à novembre ont été aussi considérés. Ces périodes ont permis d'observer clairement l'évolution des cultures pluviales, et des plans d'eau après la saison des pluies.

Les données scientifiques concernent essentiellement les paramètres climatiques qui influencent beaucoup l'environnement local. Il s'agit des évolutions des précipitations annuelles, de la longueur de la saison des pluies, de l'évolution des moyennes annuelles des températures. L'analyse de l'évolution de la moyenne annuelle des températures a concerné la période 1984 à 2013.

\section{Analyse des données statistiques}

Les données quantitatives collectées ont été traitées avec Microsoft office Excel (version 2010). Le logitiel INSTAT+ a été utilisé pour les analyses des statistiques descriptives des séries temporelles, des moyennes et des écarts types. Aussi pour déterminer certain facteur de la saison des pluies telle que la longueur de la saison des pluies.

\section{RESULTATS}

\section{La perception de la dynamique de l'occupation des terres}

Selon le constat scientifique des mutations de l'espace, l'environnement local a subi une grande modification (Figure 2). En seulement 23 ans (1992-2014), 58\% de l'environnement ont été modifiés. Ces modifications concernent essentiellement les cultures pluviales, les surfaces cuirassées, les sols nus, les plans d'eau, les savanes arborées et arbustives.

Les changements sur la dynamique de l'environnement local sont aussi observés par les populations à divers niveaux: évolution des superficies des zones dénudées, végétation, densité des arbres et arbustes, diversité des espèces.

Bien que diversement appréciée, la tendance à l'augmentation des zones dénudées est affirmée par $55 \%$ des personnes interrogées en moyenne pour les trois villages.

Ils sont plutôt nombreux, les répondants $(95 \%)$ qui trouvent que les superficies en végétation ont diminué dans leur environnement local.

La majorité des personnes enquêtées (94\%), déclarent que la densité des arbres et arbustes est en baisse.

Le même pourcentage (94\%) est observé au niveau des répondants qui constatent une diminution manifeste de la diversité des espèces végétales dans leurs 
localités. Il s'agit des espèces Ximenia americana, Coclospernum tiractorum, Planchonni lannea acida.

\section{Vision scientifique et perception des populations locales des évolutions des paramètres climatiques}

L'analyse de la Figure 3, montre une variabilité des précipitations annuelles entre la période 1985 et 2014 avec une moyenne annuelle de $672,5 \mathrm{~mm}$. En 1983, on enregistrait une précipitation annuelle de $358,2 \mathrm{~mm}$ et en 2012, les précipitations atteignaient 963,6 mm d'eau.

La Figure 4 montre aussi une variation de la longueur de la saison des pluies. On observe une tendance à la hausse croissante. Cependant, le coefficient de détermination étant en dessous du seuil de $50 \%$, la droite peut subir d'une année à l'autre une baisse.

L'évolution des moyennes annuelles des températures est très variable entre 1984 et 2013. La tendance est à l'augmentation (Figure 5).

Les analyses de la perception des populations de leur milieu ont concerné certains paramètres du climat de l'environnement local. Leurs réponses se rapportent à la variation de la pluviométrie selon le début, la fin et la durée des saisons de pluies, l'évolution de la température, et des inondations. La majorité des personnes interrogées $(81 \%)$, trouvent que le début des saisons des pluies est tardif (Figure 6).

En revanche, la fin de la saison des pluies est perçue comme précoce par la plupart des personnes enquêtées (88\%) (Figure 7).

Selon Drabo et al. (2016), les paysans au Centre-Nord du Burkina Faso perçoivent leur milieu par la baisse de la pluviométrie, la dimunition significative du nombre de pluies par an.

De l'analyse des résultats, il ressort que les paysans de l'environnement local trouvent que les températures ont augmenté ces 20 dernières années. Les résultats révèlent que
$76 \%$ d'enquêtés déclarent que la durée des périodes chaudes a augmenté ces 20 dernières années. L'augmentation des températures peut influencer la multiplication rapide des insectes avec des dégats importants sur les cultures (Habdoul Habou et al., 2016).

Les résultats de l'étude montrent que pour $52 \%$ des personnes interrogées, les impacts des variabilités pluviométriques sont surtout les inondations qui causent la destruction de cultures par asphyxie (Photo1).

\section{Les stratégies d'adaptation des populations locales}

Les paysans ne se résument pas à la description des manifestations des changements climatiques de leur environnement local, ils mettent en placent des stratégies afin de s'adapter au contexte de la variabilité climatique qui constitue un frein au développement de leurs activités agropastorales.

Les stratégies sont nombreuses, les paysans ont des critères bien donnés pour faire leur choix. Il y a la simplicité de la reproduction de la technique et aussi en termes de coût de réalisation. C'est ainsi que le zaï est très répandu dans l'environnement local. Ils sont $87 \%$ d'enquêtés qui pratiquent cette technique. La technique du zaï permet une augmentation relative du taux de matière organique qui constitue la base de la fertilité des sols notamment dégradés de la zone subsaharienne (Yaméogo et al., 2011; Somé et al., 2015). Ensuite viennent les cordons en pierres avec $85 \%$ de répondants. Le compostage est très pratiqué, $92 \%$ de personnes interrogées associent la fumure organique à l'agriculture. Les autres techniques comme les demi-lunes, les bassins de collecte, le bocage, sont moins pratiquées, etc., plus du fait de leur exigence en termes de main d'œuvre, de matériels agricoles, donc de moyens financiers que de manque de volonté de pratique. 


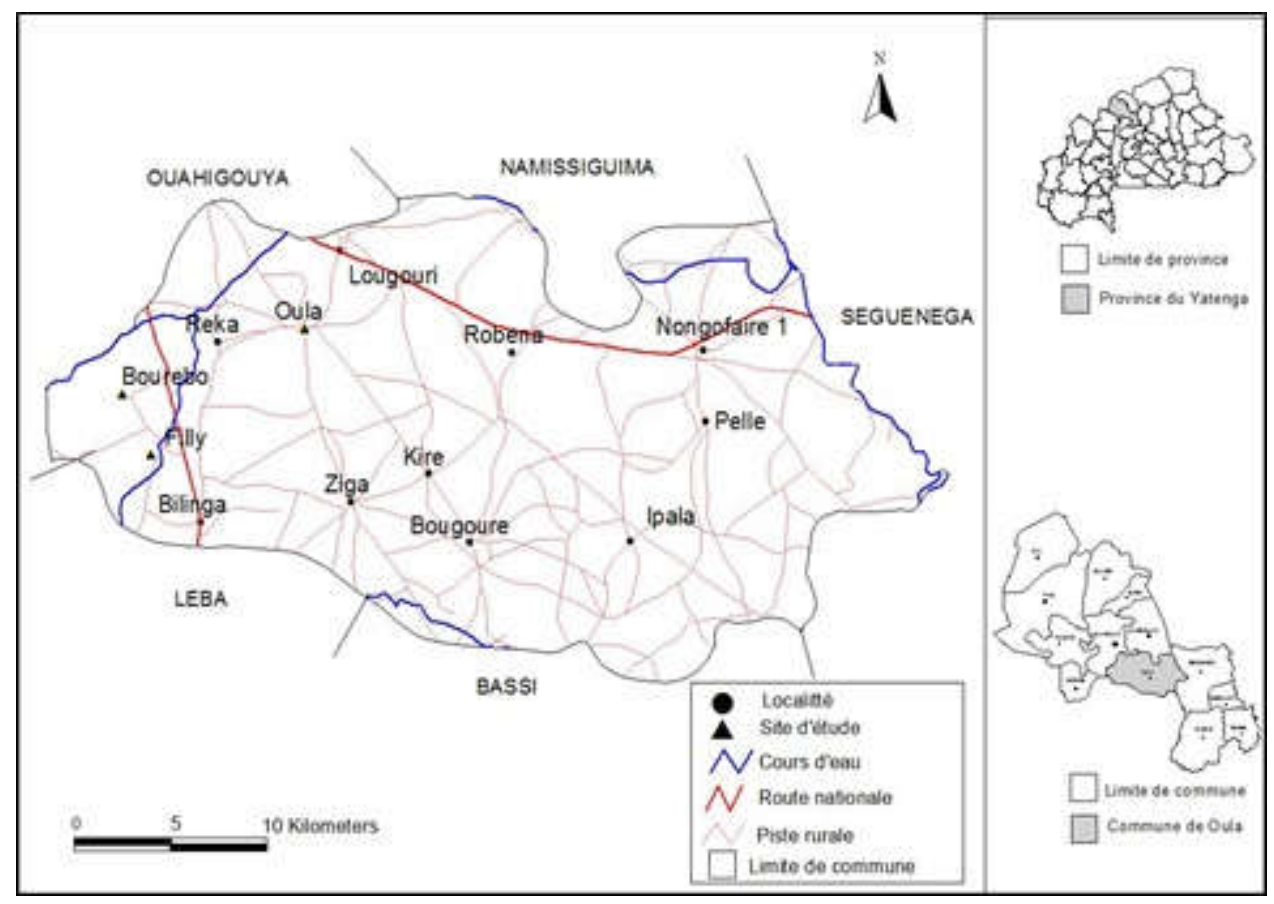

Source : IGB/BNDT 2004, Juillet 2016, OUEDRAOGO A.

Figure 1: Localisation de la zone d'étude.

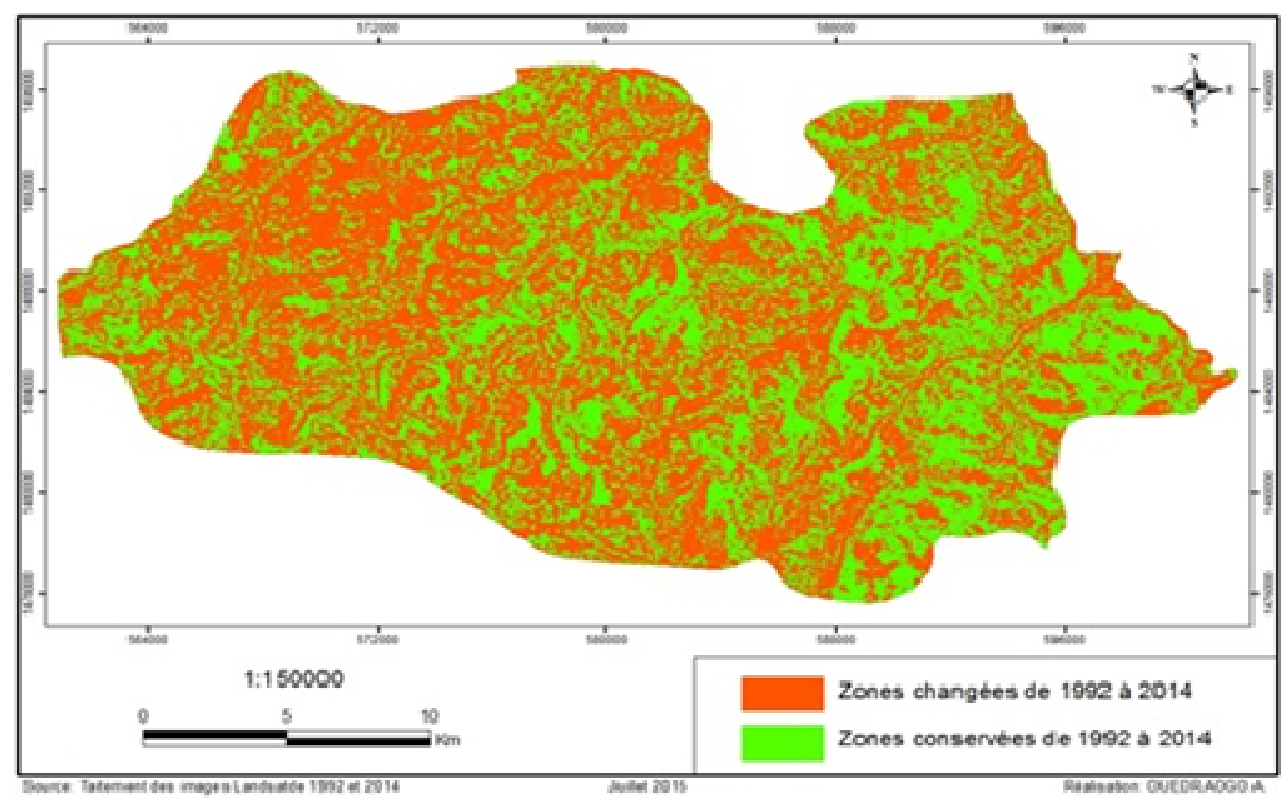

Figure 2: Mutation des espaces de l'aire d'étude entre 1992 et 2014. 


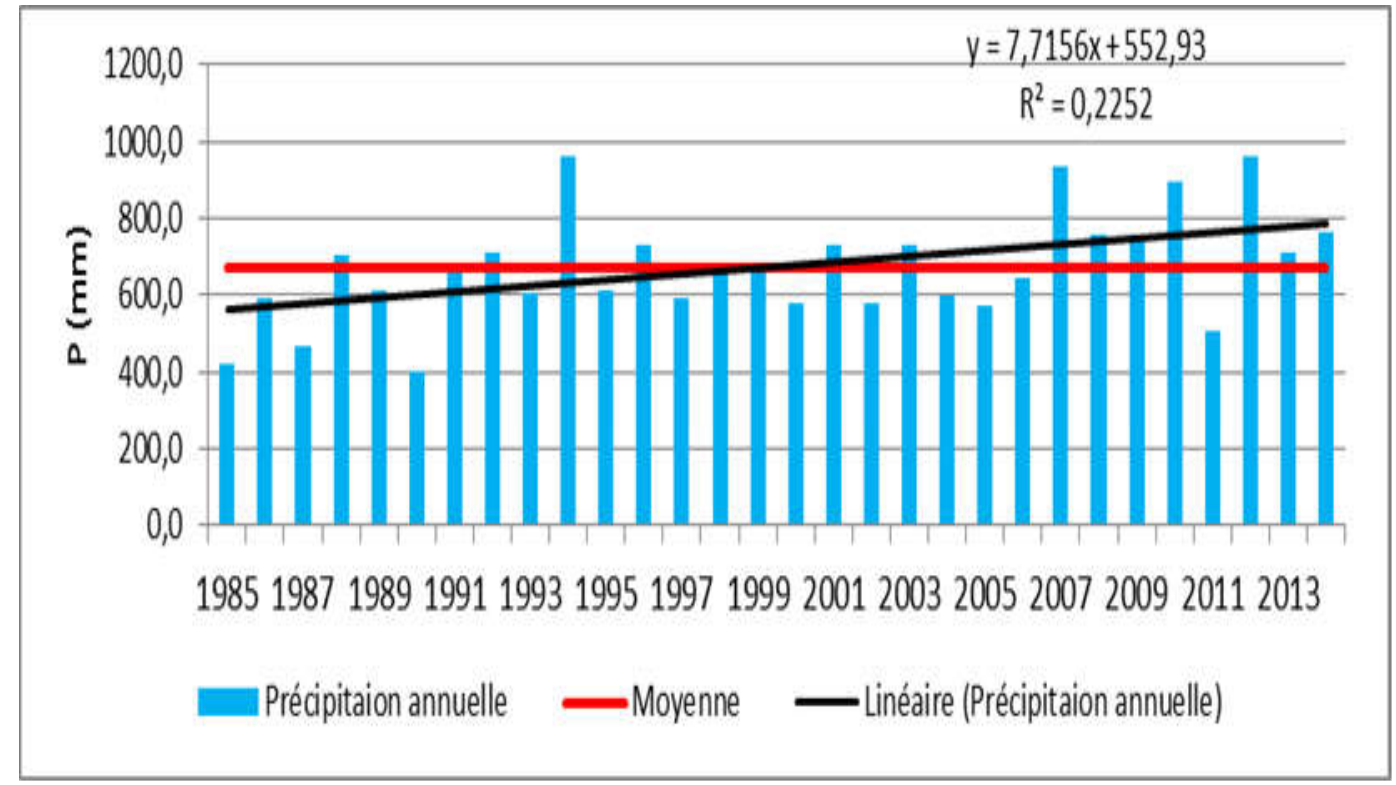

Source : Direction de la Météorologie Nationale (Burkina Faso), 2015

Figure 3: Evolution des précipitations annuelles de 1985 à 2014.

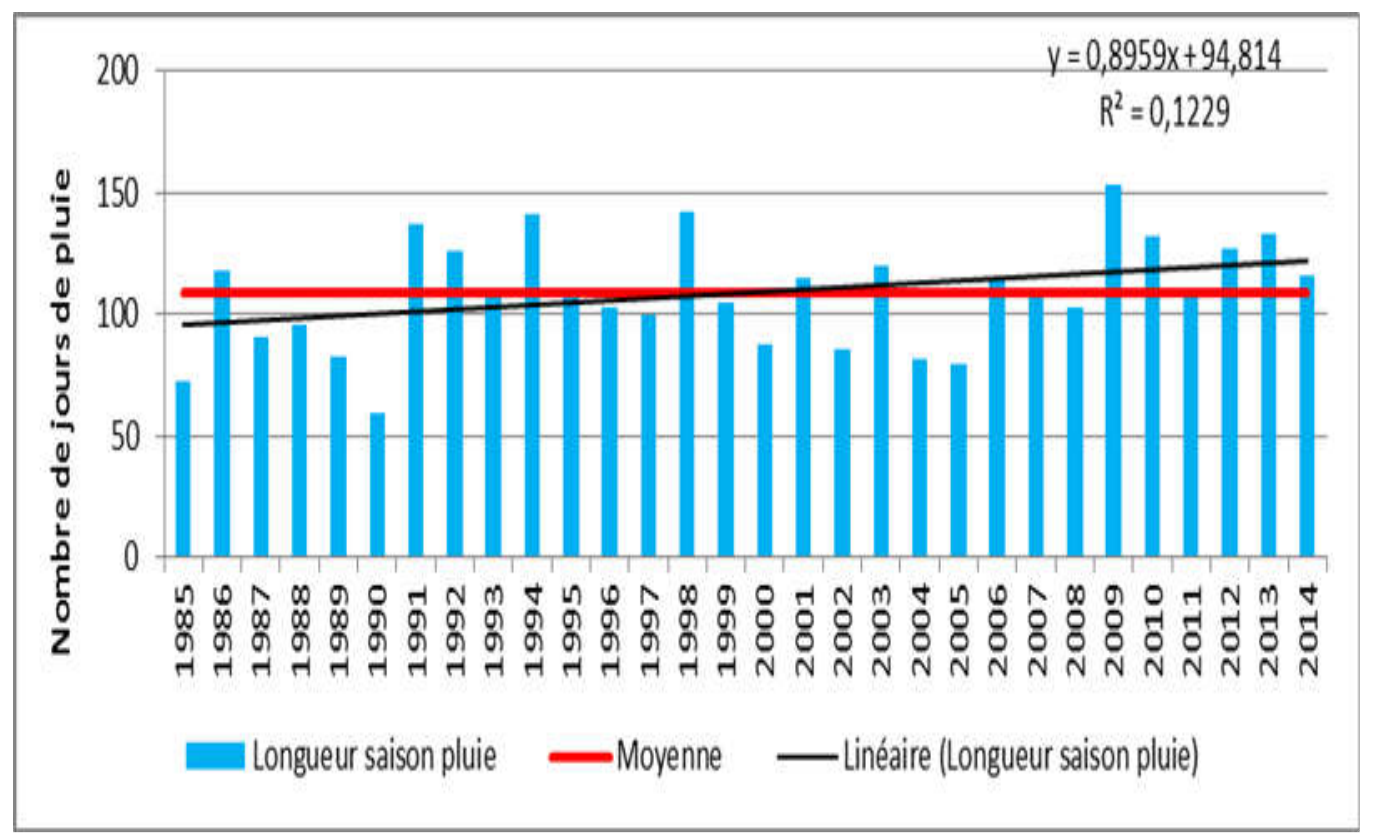

Source : Direction de la Météorologie Nationale (Burkina Faso), 2015

Figure 4: Evolution de la longueur de la saison des pluies de 1985 à 2014. 


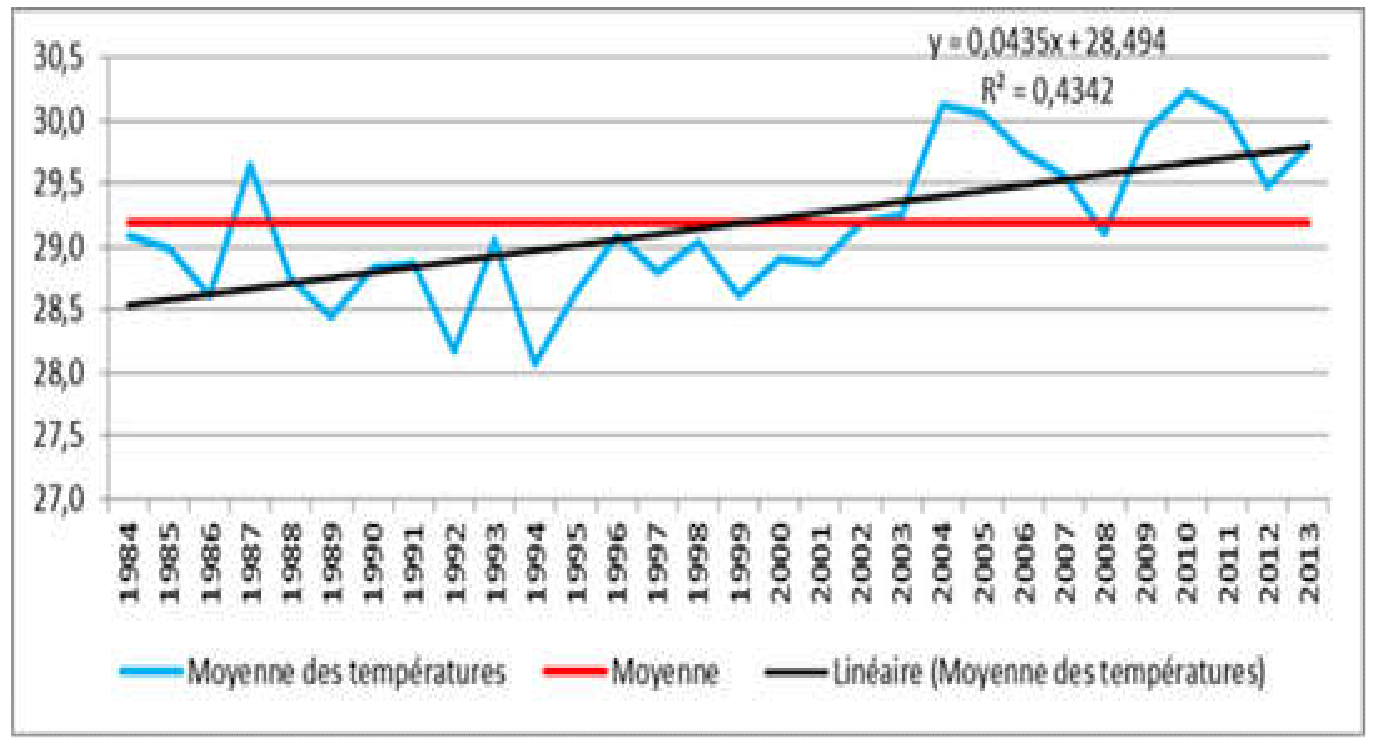

Source : Direction de la Météorologie Nationale (Burkina Faso), 2015

Figure 5: Evolution des moyennes annuelles des températures de 1984 à 2013.

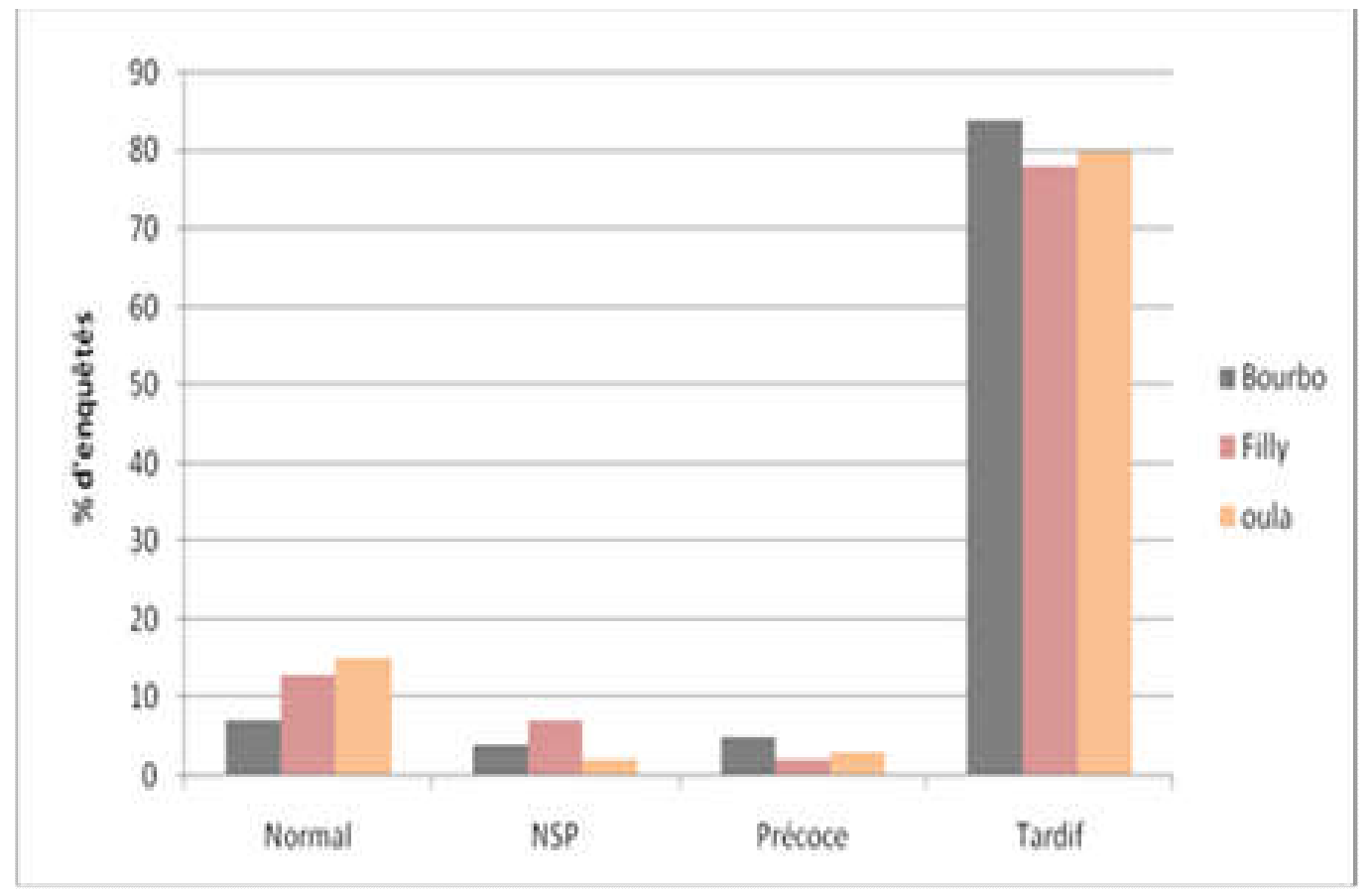

Source : Enquêtes de terrain, 2015

Figure 6: Perceptions paysannes de l'évolution du début de la saison des pluies. 


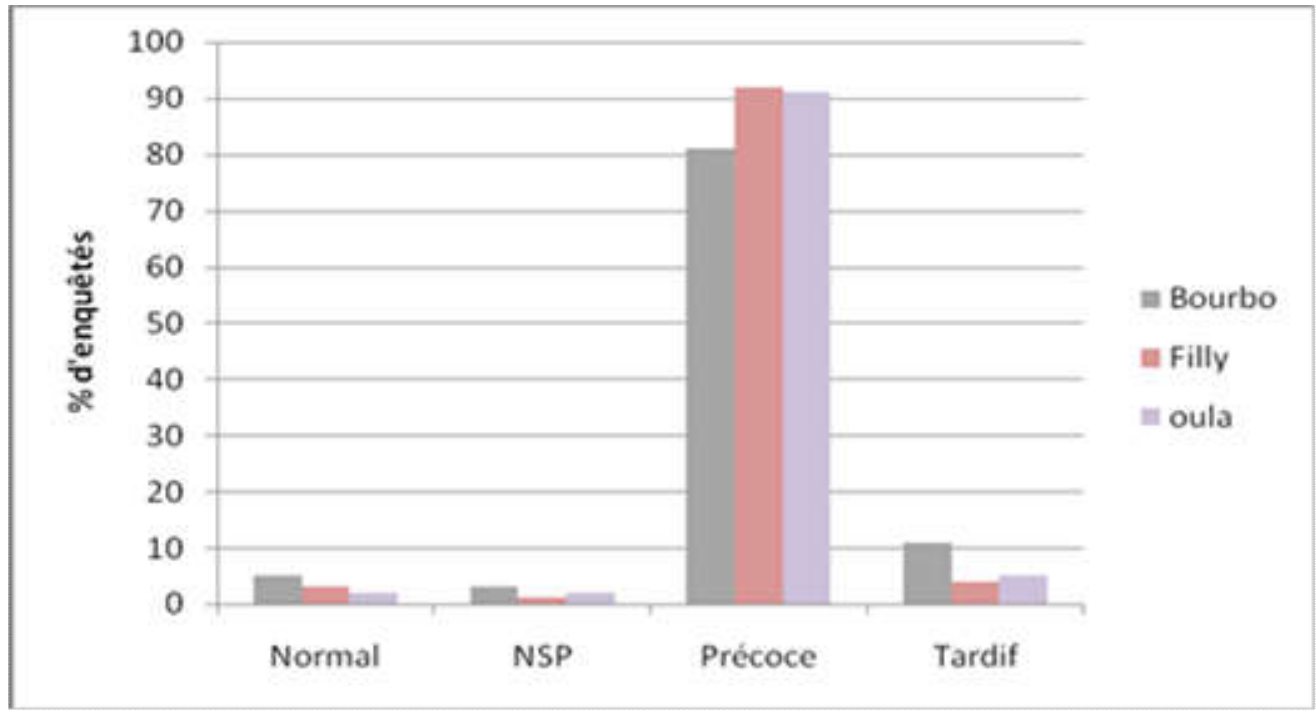

Source : Enquêtes de terrain, 2015

Figure 7: Perceptions paysannes de l'évolution de la fin de la saison des pluies.

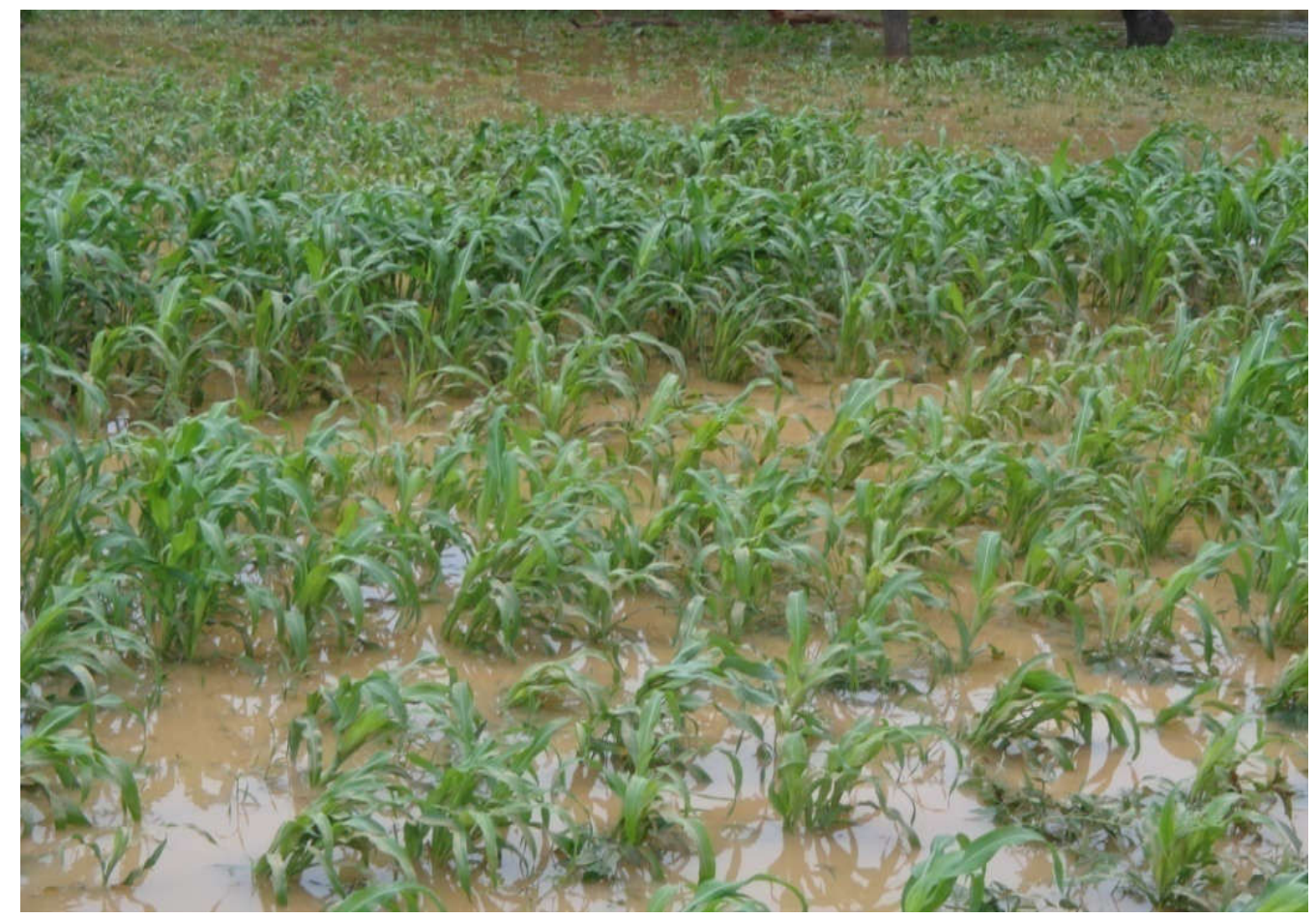

Source : Cliché terrain, août 2015

Photo 1: Champ de mil inondé par les eaux au bord du barrage (Bourbo). 


\section{DISCUSSION}

Sur la perception de la dynamique de l'occupation des terres, les paysans perçoivent clairement le changement de leur environnement. Selon Ouédraogo et al. (2010) la couverture végétale du Sahel a diminué suite aux sécheresses de 1973 et 1974. La superficie de la végétation est fortement réduite dans l'environnement local. C'est aussi l'avis des populations de la localité de Saga au Niger qui selon Touré (2013) affirmaient qu'il y a 60 à 70 ans, la végétation était très dense dans leur localité. Cette forte réduction de la superficie de la végétation s'explique par plusieurs facteurs tels que les effets des changements et variabilités climatiques mais aussi par les actions anthropiques à travers la coupe abusive du bois, les feux de brousse, la carbonisation, etc. L'augmentation des zones dénudées est due entre autres aux conséquences des sécheresses successives qu'a connu l'environnement local et aussi du fait que les techniques de cultures pratiquées contribuent à dégrader davantage les terres (Zombré, 2006).

La baisse de la densité des arbres et arbustes s'explique en partie aux effets des changements et variabilités climatiques sur l'environnement, et également à l'étalement incontrôlé des terres de culture consommatrices d'espace (Housset, 2002). La réduction de la diversité des espèces végétales est due aussi en partie aux effets des changements et variabilités climatiques sur l'environnement et le comportement des populations locales à travers les feux de brousse, la coupe du bois, les défrichements, etc.

Dans la zone d'étude, les populations locales situent le début des bouleversements des changements climatiques sur leur environnement à partir des années 1973 et 1974. Ce constat est aussi partagé par Touré
(2013), qui selon lui, les populations du Sahel estimaient que les pluies ont baissé depuis la sécheresse du début des années 1970.

Concernant le constat tardif de début de saison des pluies par les populations locales, plusieurs auteurs partagent le même point de vue. Ouoba (2013) est du même avis, dans son constat dans les villages de Dampela, Gandéfabou et Kelwélé au Nord du Burkina Faso où ces populations enquêtées affirmaient à $70 \%$ que les débuts de saison des pluies sont tardifs. Ogouwalé (2006), partage le même constat du démarrage tardif et ou les mauvaises répartitions des pluies selon $74 \%$ de personnes enquêtées dans les villages enquêtés au Bénin.

L'augmentation des températures est perçue par d'autres paysans dans différentes régions du Burkina Faso et d'Afrique. Cette observation est aussi faite par les travaux de recherche menés dans la même zone climatique (Nielsen et al., 2010).

Les inondations sont fortement ressenties par les paysans qui observent leurs champs de cultures occupés par les eaux de pluies et souvent leurs animaux domestiques sont noyés par ces eaux. Des cas similaires ont été évoqués par d'autres auteurs tels que Visser et al. (2003); Barbier et al. (2009) où les populations mentionnent des dommages liés aux pluies excessives sur la croissance des plantes au Nord du Burkina Faso.

\section{Conclusion}

Dans l'environnement local, les populations locales dans leur majorité, constatent un changement au niveau du climat. Les perceptions paysannes des changements climatiques corroborent les observations météorologiques et la littérature sur la crise climatique au Sahel. Ces changements perçus au niveau des paramètres pluviométriques et thermiques sont 
diversement appréciés. Si certaines opinions corroborent avec le constat scientifique, d'autres par contre sont contraires aux résultats des analyses météorologiques. Pour l'essentiel, le fait que les paysans soient conscients des variabilités climatiques leur confèrent un avantage dans l'élaboration des stratégies d'adaptation. En effet, la prise de conscience des variabilités climatiques ou du moins leurs effets amène les paysans à s'intéresser davantage aux techniques de lutte anti-érosive. Elles développent elles-mêmes des initiatives dans ce sens. Selon Derbile et al. (2016) les populations locales affectées par les changements et variabilités climatiques interprêtent et réagissent de manière créative pour trouver des solutions à leurs préoccupations.

S'il est vrai que les paysans pratiquent pour la plupart des activités de lutte antiérosive, leur seule capacité serait insuffisante au regard de l'état de dégradation de leur environnement surtout que la tendance est à la hausse. Aussi, les stratégies développées sontelles efficaces? Toute chose qui amène à dire qu'il faut une implication plus étroite des pouvoirs publics afin d'atteindre au mieux des résultats escomptés.

\section{CONFLIT D'INTERETS}

Il n'existe pas de conflit d'intérêts entre les différents auteurs de cet article.

\section{CONTRIBUTIONS DES AUTEURS}

$\mathrm{AO}$ a élaboré cet article. Il a participé à la lecture et à sa mise en forme. La participation à l'article peut être évaluée à hauteur de 50\%. ECDD a participé au suivi technique de la méthodologie consignée par le reviewer et aux corrections de fond et de forme de l'article. La participation peut être évaluée à hauteur de $25 \%$. APO a participé à la lecture et aux corrections de fond et de forme de l'article. La participation peut être évaluée à hauteur de $25 \%$.

\section{REMERCIEMENTS}

Les remerciements vont à l'endroit des structures techniques de l'Etat dans la Région du Nord du Burkina Faso, notamment les Directions régionales de l'agriculture, de l'élevage et de l'environnement qui ont données de précieuses informations et des conseils pratiques sur le terrain. Les remerciements vont également à l'endroit des responsables d'associations et de d'organisations villageoises, des chefs coutumiers et aux populations des villages de Bourbo, de Filly et de Oula.

\section{REFERENCES}

Barbier B, Yacouba H, Karambiri H, Zoromé M, Somé B. 2009. "Human vulnerability to climate variability in the Sahel: farmers' adaptation strategies in northern Burkina Faso". Environmental Management, 43: 790-803. DOI: http://dx.doi.org/10.1007/s00267-0089237-9.

Barrios S, Ouattara B, Strobl E. 2008. The impact of climate change on agriculture production: Is different for Africa? Food Policy, 33(4): 287-298. DOI: http://dx.doi.org/10.1016/j.foodpol.2008. 01.003 .

Derbile EK, Adams A-M, Yakubu I. 2016. Connaissance locale et évaluation communautaire des changements environnementaux au Ghana. Ghana Journal of Geography, 8(2): 59-83.

Drabo K, Yaméogo J, Sawadogo L. 2016. Examen de la gestion et des stratégies de protection des berges du Lac Bam à Kongoussi au Centre-Nord du Burkina Faso. Int. J. Biol. Chem. Sci., 10(3): 944- 
956.

DOI:

http://dx.doi.org/10.4314/ijbcs.v10i3.3.

ENEC. 2004. Enquête nationale sur les effectifs du cheptel. Ministère des ressources animales, Burkina Faso, rapport, $180 \mathrm{p}$.

GIEC. 2007. Impacts, adaptation et vulnérabilité. Contribution du Groupe de Travail II au Quatrième rapport d'évaluation du Groupe intergouvernemental sur l'évolution du climat, Cambridge, Grande Bretagne, $166 \mathrm{p}$.

GIEC. 2001. Incidences de l'évolution du climat dans les régions : évaluation de la vulnérabilité, rapport spécial, $266 \mathrm{p}$.

Habdoul Habou Z, Boubacar MK, Adam T. 2016. Les systèmes de productions agricoles au Niger face au changement climatique : défis et perspectives. Int. $J$. Biol. Chem. Sci., 10(3): 1262-1272. DOI: http://dx.doi.org/10.4314/ijbcs.v10i.3.28.

Hountondji YC, Ozer P, Nicolas J. 2004. Mise en évidence des zones touchées par la désertification par la télédétection à basse résolution au Niger. Revue Européenne de Géographie, 291: 18. DOI :

http://dx.doi.org/10.4000/cybergeo.2761.

Housset O. 2002. La dynamique régressive des espaces boisés à Tangaye. (Province du Yatenga, Burkina Faso). Université du Panthéon-Sorbonne, Maîtrise de Géographie, France, 107p.

INSD. 2008. Annuaire statistique, Ministère de l'économie et des finances, Ouagadougou, Burkina Faso, 413 p.

Mahé G, Paturel JE. 2009. Sahelian annual rainfall variability and runoff increase of Sahelian Rivers, C. R. 1896-2006. Geoscience, 341: 538-546. DOI: http://dx.doi.org/10.1016/j.crte.2009.05. 002 .
MEF 2009. Monographie de la région Nord du Burkina Faso, rapport, 184 p.

Nielsen JO, Reenberg A. 2010. Cultural barrier to climate change adaptation. A case study from Northern Burkina Faso. Global Environnent Change, 20: 142152. DOI: http://dx.doi.org/10.1016/j.gloenvcha.20 09.10.002.

Ogouwalé E. 2006. Changements climatiques dans le Bénin méridional et central : indicateurs, scénarios et prospective de la sécurité alimentaire. Thèse de Doctorat, Université d'Abomey-Calavi, Cotonou, Bénin, 302 p.

OMM. 2001. Bilan des changements climatiques 2001. Rapport de synthèse, $204 \mathrm{p}$.

Ouédraogo B. 2015. Stratégies d'adaptation des agropasteurs à la variabilité climatique dans le bassin versant de Yakouta (Burkina Faso). Thèse de doctorat unique de Géographie, Université de Ouagadougou, $257 \mathrm{p}$.

Ouédraogo M, Dembélé Y, and Somé L. 2010. Perceptions et stratégies d'adaptation aux changements des précipitations : cas des paysans du Burkina Faso. Sécheresse, Recherche, 21(2): 87-96.

Ouoba PA. 2013. Changements climatiques, dynamique de la végétation et perception paysanne dans le Sahel burkinabè (Burkina Faso). Thèse de Doctorat unique de Géographie, Département de Géographie, Université de Ouagadougou, 305 p.

Somé D, Hien E, Assigbetse K, Drevon JJ, Massé D. 2015. Dynamique des compartiments du carbone et de l'azote dans le sol cultivé en niébé et sorgho dans le système zaï en zone Nord soudanienne du Burkina Faso. Int. J. 
Biol. Chem. Sci., 9(2): 954-969. DOI: http://dx.doi.org/10.4314/ ijbcs.v9i2.32.

Tarhule A. 2011. Climate Change Adaptation in Developing Countries: Beyond. Rhetoric. Climate Variability-Some Aspects. Challenges and Prospects. Pp. 163-180.

Thornton PK, Jones PG, Ericksen PJ, Challinor AJ. 2011. Agriculture and food systems in sub-Saharan Africa in a $4{ }^{\circ} \mathrm{C}$ + world. Phil. Trans. R. Soc. A., 369: 117-136.

DOI: http://dx.doi.org/10.1098/rsta.2010.0246.

Touré A. 2013. Erosion en milieu sableux cultivé au Niger : dynamique actuelle et récente en liaison avec la pression anthropique et les changements climatiques. Thèse de Doctorat de l’Université de Bourgogne, France, 224 p.

Visser SM, Leenders JK, Leuwis M. 2003. "Farmers' perceptions of erosion by wind and water in northern Burkina Faso". Land Degradation \& Development. 14(1): $123-132 . \quad$ DOI: http://dx.doi.org/10.1002 / ldr.530.

Wittig R, König K, Schmidt M, Szarzynsky J. 2007. A study of climate change and anthropogenic impacts in West Africa, Env. Sci. Pollut. Res., 14(3): 182-189. DOI:

http://dx.doi.org/10.1065/espr2007.02.38 8.

Yaméogo JT, Hien M, Lykke AM, Somé AN, Thiombiano A. 2011. Effet des techniques de conservation des eaux et des sols, zaï forestier et cordons pierreux, sur la réhabilitation de la végétation herbacée à l'Ouest du Burkina Faso. Int. J. Biol. Chem. Sci., 5(1): 5671.

DOI : http://dx.doi.org/10.4314/ijbcs.v5i1.6808 5.

Zombré NP. 2006. Evolution de l'occupation des terres et localisation des sols nus dans le Centre-Nord du Burkina Faso. Télédétection, 5(4): 285-297.

Zoungrana BJB, Conrad C, Amekudzi LK, Thiel M, and Da ED. 2015. Land use/ cover response to rainfall variability: a comparing analysis between NDVI and EVI in the southwest of Burkina Faso. Climate, 3: 63-77. DOI. http://dx.doi.org/10.3390/cli 3010063. 